\title{
Other Scar Types: Optimal Functional and Aesthetic Outcome of Scarring in Cleft Patients
}

Wouter B. van der Sluis, Nirvana S. S. Kornmann, Robin A. Tan, and Johan P. W. Don Griot

\section{Contents}

7.1 Background $-\mathbf{5 2}$

7.2 Objectives of Cleft Lip Surgery - 52

7.3 Treatment Protocol $\mathbf{- 5 2}$

7.4 Cleft Lip Reconstruction: Surgical Techniques $\mathbf{- 5 2}$

7.4.1 Unilateral Cleft Lip - 53

7.4.2 Bilateral Cleft Lip - 53

7.4.3 Additional Measures to Improve Scarring - 53

7.5 Secondary Cleft Lip Reconstruction - 55

7.6 Evaluation of Aesthetic Outcome - $\mathbf{5 5}$

7.6.1 Palatal Scarring - 55

7.7 Conclusion -56

Further Reading - 57 


\subsection{Background}

Cleft lip and palate are facial and oral malformation due to failures in the embryologic craniofacial development during early pregnancy. They occur in approximately 1 in 750/1000 births in Europe, which makes it the most common congenital craniofacial abnormality. Different variations in phenotype of this malformation exist. It can occur unilateral (left or right) or bilateral, with involvement of lip and/or alveolus and/or palate and can be microform, incomplete, or complete. Unilateral cleft lip and palate are the most common type, whereby the upper lip, the orbicularis muscle, the alveolar bone, the floor of the nose, and the hard and soft palate are interrupted, creating an open communication between nasopharynx and oropharynx.

Patients with a cleft lip and palate are treated in specialized cleft centers by a multidisciplinary team, consisting of geneticists; (plastic) surgeons; orthodontists; dentists; ear, nose, and throat (ENT) doctors; speech and language therapists; and psychologists. All surgical interventions aimed at closure of the cleft lip and palate induce restriction of maxillary growth. Surgical timing, staging, and techniques differ between treatment centers. The lip is usually closed at 3-6 months after birth and the soft palate at 6-12 months. In some cleft centers, the hard palate is closed before 12 months after birth in either a one-stage or two-stage procedure. Other cleft centers prefer to close the hard palate later in life, at 18 months to 3 years, or during the closure of the alveolar gap at the age of $8-11$ years.

Having cleft lip and/or palate has a noteworthy impact on quality of life and psychosocial functioning. Postoperative scarring is a common cause of patient dissatisfaction. The degree of postoperative scarring in cleft patients is associated with symptoms of anxiety, depression, and a lower self-esteem. For cleft surgeons, every possible measure to optimize functional and aesthetic outcome is important.

\subsection{Objectives of Cleft Lip Surgery}

The goal of cleft lip surgery is to close the lip separation, provide optimal function in terms of speech, mastication, dental protection, breathing and feeding, and provide an aesthetically pleasing facial scar and optimal shape of nose and nostril. The visible facial scar and its effect on nose and lip aesthetics are a daily reminder to the patient of its underlying cause. Over the years, different surgical techniques have been described regarding surgical closure of the cleft lip. The ideal technique should create a balanced lip, allow for easy adjustments, and produce a favorable scar pattern combined with a
- Table 7.1 Short overview of the "surgical treatment of cleft patients" protocol of the Amsterdam University Medical Center

\begin{tabular}{|c|c|c|}
\hline $\begin{array}{l}\text { Unilateral cleft lip } \\
\text { and palate }\end{array}$ & $\begin{array}{l}\text { Bilateral cleft lip } \\
\text { and palate }\end{array}$ & Cleft palate \\
\hline $\begin{array}{l}3 \text { months: Lip } \\
\text { closure }\end{array}$ & $\begin{array}{l}3 \text { months: Lip } \\
\text { closure }\end{array}$ & \\
\hline $\begin{array}{l}9 \text { months: } \\
\text { Palatography }\end{array}$ & $\begin{array}{l}9 \text { months: } \\
\text { Palatography }\end{array}$ & $\begin{array}{l}9 \text { months: } \\
\text { Palatography }\end{array}$ \\
\hline $\begin{array}{l}\text { 7-9 years: } \\
\text { If necessary, } \\
\text { hard palate } \\
\text { closure } \\
\text { If necessary, } \\
\text { orthognathic } \\
\text { surgery }\end{array}$ & $\begin{array}{l}\text { 7-9 years: } \\
\text { If necessary, } \\
\text { hard palate } \\
\text { closure } \\
\text { if necessary, } \\
\text { orthognathic } \\
\text { surgery }\end{array}$ & $\begin{array}{l}\text { 7-9 years: Hard } \\
\text { palate closure }\end{array}$ \\
\hline $\begin{array}{l}>15 \text { years } \\
\text { If necessary, } \\
\text { secondary } \\
\text { corrections }\end{array}$ & $\begin{array}{l}>15 \text { years } \\
\text { If necessary, } \\
\text { secondary } \\
\text { corrections }\end{array}$ & $\begin{array}{l}>15 \text { years } \\
\text { If necessary, } \\
\text { secondary } \\
\text { corrections }\end{array}$ \\
\hline $\begin{array}{l}>17 \text { years } \\
\text { If necessary, } \\
\text { rhinoplasty }\end{array}$ & $\begin{array}{l}>17 \text { years } \\
\text { If necessary, } \\
\text { rhinoplasty }\end{array}$ & \\
\hline
\end{tabular}

symmetrical shape of the nose. Closure of a cleft lip, also called "cheilorrhaphy," is a functional and aesthetic reconstruction of the upper lip, orbicularis muscle, nasal floor, and ala.

\subsection{Treatment Protocol}

As stated above, surgical timing, staging, and techniques differ between treatment centers. In $\bullet$ Table 7.1, a short overview of the "surgical treatment of cleft patients" protocol of the Amsterdam University Medical Center is provided.

\subsection{Cleft Lip Reconstruction: Surgical Techniques}

Precise surgical technique and adequate aligning of anatomical structures is important for the postoperative aesthetic result and scar formation. Patients with a cleft lip have, besides the obvious interrupted upper lip, a typical flared nostril and a septum deviation. As stated above, different surgical techniques are used to reconstruct this defect, each with its pros and cons. Though many approaches exist, the Millard (rotation advancement) and Fisher (anatomic subunit) approaches are historically most frequently performed. 


\subsubsection{Unilateral Cleft Lip}

\subsubsection{Millard Lip Closure}

Since the 1950s, the Millard rotation advancement technique for surgical lip closure was the most popular among cleft surgeons. It consists of downward rotation of the medial cleft component and a lateral lip advancement flap. The majority of the scar can be placed at the philtral column. A disadvantage of this approach is that the superior part of the scar crosses the philtrum perpendicularly at the columellar base. Various modifications of the traditional Millard technique have been developed to overcome this disadvantage.

\subsubsection{Fisher Lip Closure}

In 2005, Fisher published his lip closure technique, in which the anatomical subunit principles guide lip repair. In this technique, a circumferential incision at the cleft side of the columella is continued to the Cupid's bow top in a straight manner. This mirrors the unaffected philtral ridge. To lengthen the medial flap, small inlet incisions are frequently necessary. To prevent upper lip shortening, a triangular flap above the cutaneous roll can be incorporated (- Figs. 7.1, 7.2, and 7.3).

\subsubsection{Bilateral Cleft Lip}

"Bilateral cleft lip" can manifest in different ways, in which symmetrical complete bilateral

cleft lip-cleft palate is the most prevalent. When looking at the lip alone, the bilateral symmetrical complete variant accounts for $50 \%$ of cases, bilateral incomplete for $25 \%$, and asymmetrical bilateral (complete/ incomplete) for $25 \%$. A one-stage surgical approach is advocated in most centers.

The Millard-Mulliken technique is frequently employed. A cranially based philtrum flap is created, the gap is closed, and orbicularis oris continuity and lip continuity are restored. Ideally, both scars are positioned symmetrically at the philtral ridge.

In several cleft centers these surgical techniques are combined with preoperative nasoalveolar molding (NAM). The aim of NAM is to improve the shape and form of the alveolar wall and nose and to bring the divided lip together with an orthodontic plate, nasal spring, and tape (Plaatje). In our center NAM is only used in cases with bilateral cleft lips. Furthermore, a nasal conformer is used in unilateral cases to improve the shape of the affected nostril postoperative.

\subsubsection{Additional Measures to Improve Scarring}

Visible facial scarring after cleft lip repair is inevitable. There are however some perioperative measures that can be taken to minimize scar morbidity in terms of hypertrophy and contraction.

\subsubsection{Perioperative Botulinum Toxin}

After surgical correction of the lip and philtrum, the underlying orbicularis oris muscle contracts in a direction perpendicular to the cutaneous scar. This results in accentuation of dynamic rhytides, which may slowly result in widening of the surgical scar. Some surgical teams temporarily paralyze the orbicularis oris muscle, by intraoperatively injecting it with botulinum toxin. Several studies showed a beneficial effect on postoperative scar width; however, the sample size is relatively small in most studies, and objective assessment tools are scarcely employed.

\subsubsection{Sutureless Skin Closure}

Using (too many) cutaneous sutures in a facial wound may cause a foreign body reaction and subsequent suboptimal scarring. The possibility of visible crosshatching of sutures is unappealing.
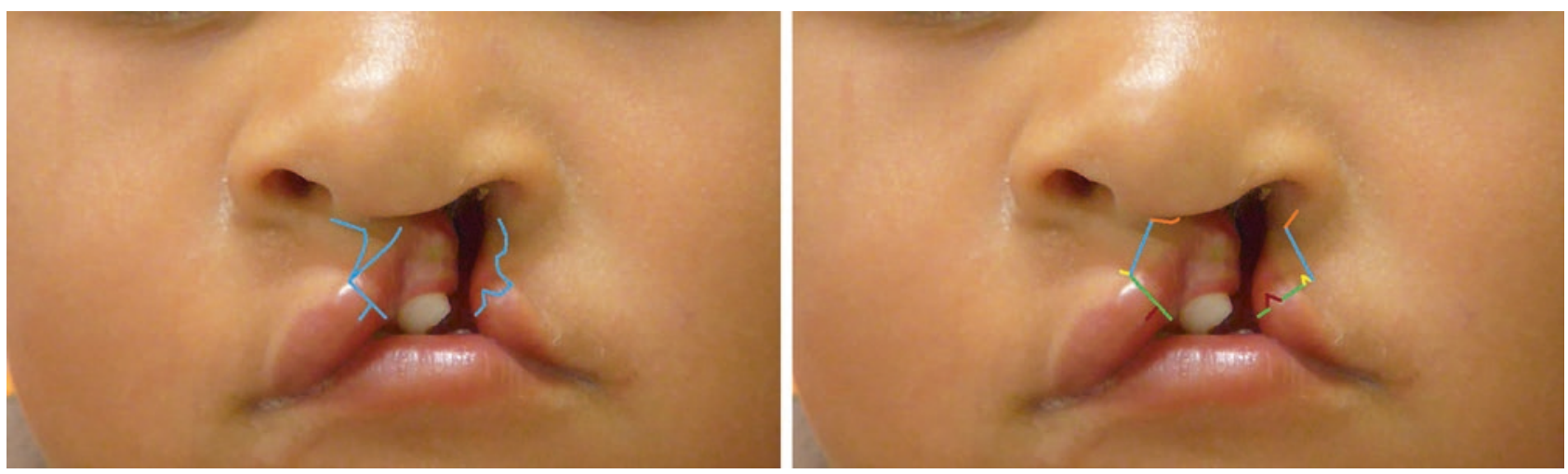

Fig. 7.1 Preoperative planning for Millard (left) and Fisher (right) lip closure 

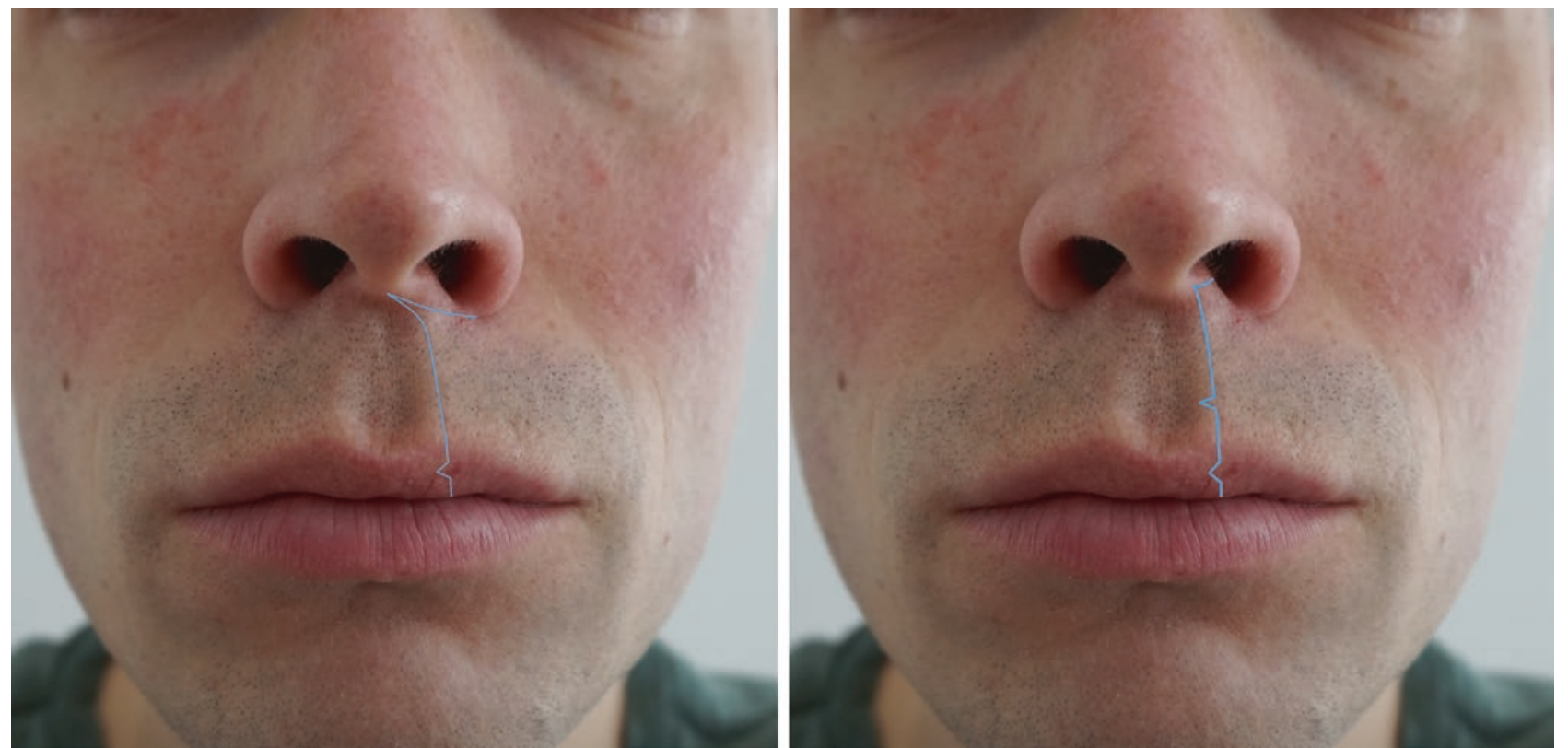

- Fig. 7.2 Postoperative scar pattern after Millard (left) and Fisher (right) lip closure. Note, different surgeons use different modifications of both techniques

- Fig. 7.3 Preoperative (upper), planning (middle), and direct postoperative (lower) result of the Fisher lip closing technique in a patient with complete unilateral cleft lip and palate
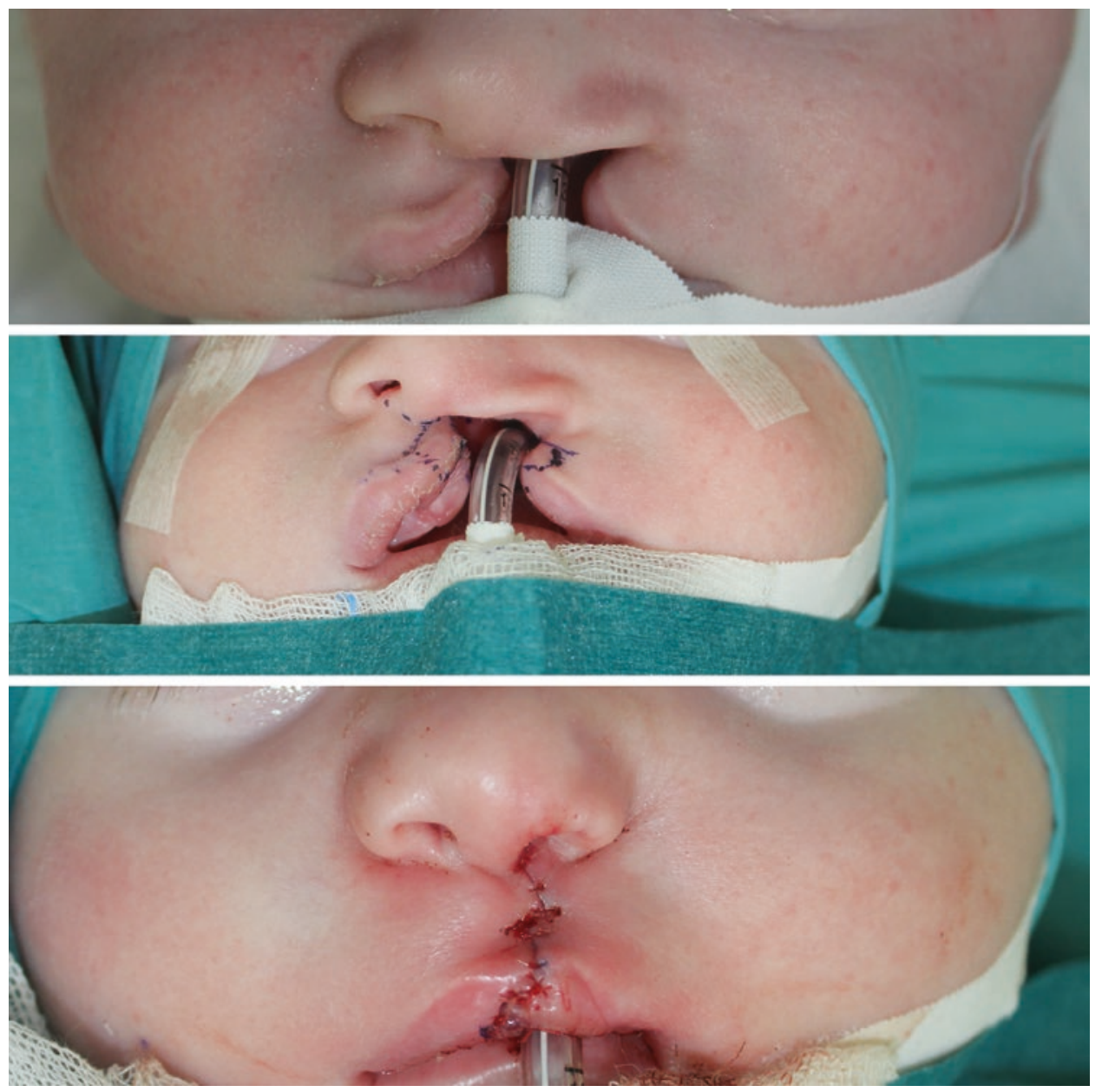
Some advocate the use of sutureless skin closure, in which the cutaneous layer approximation is performed using surgical glue, such as butyl cyanoacrylate or amcrylate. An additional advantage is that is generally faster to perform, which reduces intraoperative time. No large, prospective comparative studies are performed on this subject.

\subsubsection{Silicone Application}

Topical silicone application is used for treating/preventing hypertrophic scars in different body locations. This is also the case for cheiloplasty scars. To which extent hypertrophic cheiloplasty scars can be treated/prevented with this application is unknown. Side effects of this treatment comprise skin rash and fusibility problems, such as loss of adhesiveness. Using silicone gel seems to be non-inferior to silicone sheeting.

\subsubsection{Postoperative Laser Therapy}

Use of carbon dioxide fractional laser, sometimes combined with intense pulsed light treatment, in the direct postoperative phase is shown to improve scars in terms of pliability and color. Though proven safe, pain during the session and crust formation are frequently reported.

\subsubsection{Hair Transplantation}

In male cleft patients, limited or missing moustache growth may be noticeable in the scarred area, especially in men with dark hair and ample hair growth. Hair graft transplantation can be performed; however, the poor vascularization of scar tissue poses a problem, because this is not a good bed for graft survival. Some use fat grafting of the scar before hair transplantation to optimize graft survival.

\subsection{Secondary Cleft Lip Reconstruction}

Secondary cleft lip reconstruction is frequently performed due to scar contracture, which has a negative impact on lip, philtrum, and nose aesthetics. The exact incidence of secondary lip correction is unknown and dependent on many factors, for example, patient factors, treatment protocol, access to health care services, etc. In the Western world, the estimated incidence is $40-60 \%$. Conservative management of these cases is seldom successful. Surgical correction typically employs use of the Rose-Thompson effect (in which a scar is lengthened by using concave excisions of the scar, which is subsequently closed in a straight line), one or multiple Z-plasties, or a combination of these strategies. Some perform fat grafting of the scar to loosen it and provide more length. Remaining volume deficiencies, for exam- ple, volume deficiencies of the lip, can be restored in the same procedure with lipofilling or dermal fat grafts.

\subsection{Evaluation of Aesthetic Outcome}

One of the goals in the treatment of cleft lip (and palate) repair is improving nasolabial aesthetics by restoring symmetry and proportion of the nose and lip. Traditionally, aesthetic assessment is performed by symmetry measurements with a caliper. The last two decades, several scoring systems have been proposed for a simpler and quicker assessment. Most of these scoring systems use two-dimensional (2D) photographs in combination with Likert or visual analog (VAS) scales. The most frequently used method is the Asher-McDade (AMD) system, which utilizes a five-point Likert scale ranging from "excellent" to "very poor" to grade four anatomical nasal and lip structures. In 2016, the Cleft Aesthetic Rating Scale (CARS) was developed as a new tool to allow rapid assessment of the nose and lip separately.

The majority of assessment methods are focusing on symmetry and on the shape of the nose and lip, whereas the scar appears to be of secondary importance. In 2010, the Patient and Observer Scar Assessment Scale (POSAS) was proposed to assess the scar after cleft lip repair. Nevertheless, it remains unknown to which extend visible scarring plays a role in facial aesthetics after cleft surgery.

To determine the aesthetic outcome in a more objective way, measurements using three-dimensional (3D) photographs seem to be promising nowadays. These methods show a high reliability, but these techniques require expertise and costly equipment and are therefore not readily available worldwide. To compromise, computer-based programs, like SymNose, have been developed. These programs allow symmetry measurements on 2D images of the nose and lip by tracing the outline of the upper lip and the lower border of the nose. By reflecting the left side of the midline over the right, the percentage mismatch of the nonoverlapping area is calculated.

However, an internationally accepted assessment method for the aesthetic evaluation of cleft lips is still not available.

\subsubsection{Palatal Scarring}

\subsubsection{Palatal Closure}

Both the hard palate and soft palate can be involved in palatal clefts, resulting in a gap from anterior to posterior, affecting the maxilla, the mucosa, the levator veli 
palatini muscle, the tensor veli palatini muscle, and uvula muscle. An open communication between the nasopharynx and oropharynx prevents the infant to create an intraoral negative pressure, which is mandatory for productive suckling during (breast)feeding. A positive oropharyngeal pressure and elevation of the palate allows for the normal articulation of the oral consonants, most notably the oral stop-plosives, [p, t, k, b, d, g] in English. This can only be achieved by partitioning the oropharynx from the nasopharynx. Therefore, the aim of palatal closure, also called "palatography" or "palatoplasty," is functional reconstruction of structures that are necessary for feeding and speech development early in life. Different types of palatoplasties have been described to close the soft and the hard palate whereby several surgical considerations must be addressed. The most important anatomical structure, which should not be harmed during surgery is the greater palatine neurovascular bundle, proceeding through the greater palatine foramen through the lateral posterior hard palate. It is essential to obtain a tension-free closure of the palatal flaps, preventing compression or stretching of the neurovascular bundle. Releasing incisions may be necessary to achieve complete closure from anterior to posterior.

\subsubsection{Timing of Palatal Closure}

Impaired maxillary growth and an abnormal speech development are common findings in

patients after repaired cleft lip and palate. Delayed closure of the palate beyond 12 years or no closure at all minimizes abnormal facial growth, yet early closure of the palate, that is, before 12 months after birth, is necessary for normal speech development. Studies on speech development, maxillary growth, and timing of palatal closure show different results due to confounding variables as surgical technique, surgeon's experience level, and lack of standardized speech outcome or standardized indications for secondary maxillary surgery. Therefore, consensus on the optimal timing of palatal closure has not yet been reached.

\subsubsection{Maxillary Growth}

Impaired maxillary growth in cleft patients often results in typical features as crowding, lateral crossbite, and open bite. The exact pathophysiology of abnormal facial growth after cleft lip and palate repair remains unclear. It is a widely accepted hypothesis that abnormal maxillary growth has an iatrogenic cause and is secondary to surgical intervention due to scarring. However, other studies suggest that intrinsic maxillary underdevelopment contribute to impaired facial growth as well. It is most likely that a combination of intrinsic and iatrogenic factors is responsible, making it mandatory for a surgeon to minimize scarring in the oral cavity.

\subsubsection{Speech Development}

Due to a congenital short palate, hypernasal speech, and nasal air emission during speech are common findings in children after a cleft palate repair. Hypernasality and nasal air leakage are often the result of velopharyngeal insufficiency, which means that the velopharyngeal valve does not close completely and consistently during the production of oral sounds. As scarring tissue tends to contract in longitudinal direction, the palate frequently becomes even shorter or movement of the soft palate becomes inadequate, preventing the soft palate to make contact with the pharyngeal wall. Several techniques have been described to create more length of the palate, such as the V-Y pushback repair (Veau-WardillKilner), the buccal mucosal flap, the double opposing Z-plasty (Furlow), or the lengthening of the soft palate with a pharyngoplasty.

\subsection{Conclusion}

Cleft lip and palate scars influence lip, philtrum, and nose aesthetics, as well as speech and growth of the maxilla. Optimal scar management can be divided in surgical (precise surgical technique, planning, and adequate aligning of anatomical structures) and nonsurgical methods (botulinum toxin, silicone application, carbon dioxide fractional laser).

\section{Take-Home Message}

- Cleft lip and palate are facial and oral malformation due to failures in the embryologic craniofacial development during early pregnancy.

- The goal of cleft lip surgery is to close the lip separation; provide optimal function in terms of speech, mastication, dental protection, breathing and feeding; and provide an aesthetically pleasing facial scar.

- Cleft lip scars influence lip, philtrum, and nose aesthetics.

- Optimal scar management can be divided in surgical (precise surgical technique, planning, and adequate aligning of anatomical structures).

- And nonsurgical methods (botulinum toxin, silicone application, carbon dioxide fractional laser).

- In secondary lip correction, cleft surgeons typically make use of the Rose-Thompson effect, one or multiple Z-plasties, or a combination of these strategies. 


\section{Further Reading}

Fu KJ, Teichgraeber JF, Greives MR. Botulinum toxin use in pediatric plastic surgery. Ann Plast Surg. 2016;77(5):577-82.

Chang CS, Wallace CG, Hsiao YC, Huang JJ, Chen ZC, Chang CJ, Lo LJ, Chen PK, Chen JP, Chen YR. Clinical evaluation of silicone gel in the treatment of cleft lip scars. Sci Rep. 2018;8(1):7422.

Fisher DM. Unilateral cleft lip repair: an anatomical subunit approximation technique. Plast Reconstr Surg. 2005;116(1): 61-71.

Millard DR. Refinements in rotation advancement cleft lip technique. Plast Reconstr Surg. 1964;33:26-38.

Akdag O, Evin N, Karamese M, Tosun Z. Camouflaging cleft lip scar using follicular unit extraction hair transplantation combined with autologous fat grafting. Plast Reconstr Surg. 2018;141(1):148-51.
Akdag O, Evin N, Karamese M, Tosun Z. Camouflaging cleft lip scar using follicular unit extraction hair transplantation combined with autologous fat grafting. Plast Reconstr Surg. 2018;141(1):148-51.

Mosmuller DGM, Mennes LM, Prahl C, Kramer GJC, Disse MA, van Couwelaar GM, Niessen FB, Griot JPWD. The development of the cleft aesthetic rating scale: a new rating scale for the assessment of nasolabial appearance in complete unilateral cleft lip and palate patients. Cleft Palate Craniofac J. 2017;54(5): 555-61.

Pigott RW, Pigott BB. Quantitative measurement of symmetry from photographs following surgery for unilateral cleft lip and palate. Cleft Palate Craniofac J. 2010;47(4):363-7.

Open Access This chapter is licensed under the terms of the Creative Commons Attribution 4.0 International License (http://creativecommons. org/licenses/by/4.0/), which permits use, sharing, adaptation, distribution and reproduction in any medium or format, as long as you give appropriate credit to the original author(s) and the source, provide a link to the Creative Commons license and indicate if changes were made.

The images or other third party material in this chapter are included in the chapter's Creative Commons license, unless indicated otherwise in a credit line to the material. If material is not included in the chapter's Creative Commons license and your intended use is not permitted by statutory regulation or exceeds the permitted use, you will need to obtain permission directly from the copyright holder. 